\title{
Proteolytic cleavages of MET: the divide-and-conquer strategy of a receptor tyrosine kinase
}

\author{
Marie Fernandes ${ }^{\#}$, Leslie Duplaquet" $\&$ David Tulasne* \\ University of Lille, CNRS, Institut Pasteur de Lille, UMR 8161 - M3T - Mechanisms of Tumorigenesis and Target Therapies, F-59000 Lille, \\ France
}

\begin{abstract}
Membrane-anchored full-length MET stimulated by its ligand $\mathrm{HGF} / \mathrm{SF}$ induces various biological responses, including survival, growth, and invasion. This panel of responses, referred to invasive growth, is required for embryogenesis and tissue regeneration in adults. On the contrary, MET deregulation is associated with tumorigenesis in many kinds of cancer. In addition to its well-documented ligand-stimulated downstream signaling, the receptor can be cleaved by proteases such as secretases, caspases, and calpains. These cleavages are involved either in MET receptor inactivation or, more interestingly, in generating active fragments that can modify cell fate. For instance, MET fragments can promote cell death or invasion. Given a large number of proteases capable of cleaving MET, this receptor appears as a prototype of proteolytic-cleavage-regulated receptor tyrosine kinase. In this review, we describe and discuss the mechanisms and consequences, both physiological and pathological, of MET proteolytic cleavages. [BMB Reports 2019; 52(4): 239-249]
\end{abstract}

\section{INTRODUCTION}

MET is a receptor tyrosine kinase (RTK) produced predominantly in cells of epithelial origin. It is activated by its stromal ligand, the hepatocyte growth factor (HGF), also called as scatter factor (1). HGF and MET are essential for embryonic development: their knockout affects epithelial organ, placenta, muscle, and neuron formation (2-5). In adults, the HGF-MET pair is involved in physiological processes such as epidermal healing and liver regeneration (6-8).

MET is synthesized as a precursor that matures through

${ }^{*}$ Corresponding author. Tel: +33-3-20-87-12-57; Fax: +33-3-20-87 11-11; E-mail: david.tulasne@ibl.cnrs.fr

${ }^{\#}$ These authors contributed equally to this work.

https://doi.org/10.5483/BMBRep.2019.52.4.024

Received 9 January 2019

Keywords: Apoptosis, Calpain, Caspase, Hepatocyte growth factor, MET, Necrosis, Protease, Proteolytic cleavages, Receptor tyrosine kinase, Secretase proteolytic cleavage between amino acids 307 and 308 by furin. The cleavage leads to the formation of a heterodimeric receptor composed of an extracellular $\alpha$ subunit (50 kDa) linked via a disulfide bond to a single-pass transmembrane $\beta$ subunit (145 kDa). The extracellular part of MET contains an $\mathrm{N}$-terminal SEMA domain encompassing an $\alpha$ subunit and the first amino acids of the $\beta$ subunit, followed by a plexinsemaphorin-integrin domain and four immunoglobulin-like domains.

The intracellular region of MET contains a juxtamembrane domain, the tyrosine kinase domain, and a C-terminal tail (9). The interaction of MET with its ligand favors receptor dimerization and autophosphorylation of two tyrosine residues present in the catalytic domain (Y1234 and Y1235). Subsequently, other tyrosine residues, located outside the kinase domain get phosphorylated, particularly tyrosine 1003 in the juxtamembrane domain and tyrosines 1349 and 1356 in the C-terminal tail $(10,11)$. The phosphorylated C-terminal residues allow recruitment of important effectors such as GAB1, GRB2, SHC, PI3K, SRC, STAT3, and PLC $\gamma$. This multi-substrate docking site plays a key role in MET-induced biological responses and its mutation in mice triggers phenotypes similar to those of MET-deficient mice (12).

In parallel, downregulation of MET receptor activity is an essential process which prevents receptor oversignaling. Interestingly, all the regulatory mechanisms evidenced so far are located in the juxtamembrane region of the cytoplasmic part of the receptor, encoded by exon 14 . First, in response to HGF/SF, MET phosphorylation at tyrosine residue 1003 leads to recruitment of the ubiquitin ligase $\mathrm{CBL}$, leading to ubiquitination and lysosomal degradation of the receptor (13). Second, ligand stimulation induces phosphorylation of serine residue 985, which is involved in downregulation of MET kinase activity (14).

Aberrant HGF-MET signaling is involved in tumorigenesis and metastasis (for review, see 15). In a significant number of human cancers, overexpression of the genes encoding HGF and MET causes either paracrine or autocrine activation of the receptor and is associated with poor prognosis (16-22). Massive synthesis of MET, leading to its ligand-independent activation is notably related to amplification of the MET gene, and the phenomenon was observed in a small percentage 
(about 1 to $5 \%$ ) of gastric and lung cancers (23-25). Numerous MET mutations have been described in various cancer types, including renal and lung cancers (26). In renal cancers, MET mutations are located mainly in the kinase domain, causing constitutive kinase activity. In lung cancers, MET mutations affect the juxtamembrane domain in about $3 \%$ of patients (27). Notably, various mutations impair the splice acceptor and donor sites of exon 14, causing exon skipping and deletion of a large part of the juxtamembrane domain. Deletion of the juxtamembrane domain favors receptor activation by its ligand since this domain displays several negative regulatory sites (28).

\section{PROTEOLYTIC CLEAVAGES OF MET}

\section{Secretase cleavages of MET: regulation of receptor half-life}

The first MET fragments were detected in the early 1990s with monoclonal antibodies directed against the extracellular part of the MET $\beta$ chain. These antibodies revealed in a culture supernatant an $\mathrm{N}$-terminal fragment of about $90 \mathrm{kDa}$ (MET-NTF) $(29,30)$. Later, the likely C-terminal counterpart was described as an unstable $55 \mathrm{kDa}$ intracellular fragment (31). Actually, these fragments are generated by the first step of the proteolytic process called presenilin-regulated intramembrane proteolysis (PS-RIP). This process involves initial cleavage by membrane metalloproteases, causing MET to shed its extracellular domain at an unidentified cleavage site likely located near the transmembrane domain. This shedding seems to involve the ADAM metalloproteases 10 and 17, as shown by its inhibition through silencing of the corresponding genes $(32,33)$. This initial cleavage generates both, the $\mathrm{N}$-terminal fragment MET-NTF (also called soluble MET, sMET), which is released into the extracellular space and the membraneanchored C-terminal fragment MET-CTF (29-31, 34) (Fig. 1A). This proteolytic process is immediately followed by a second cleavage of MET-CTF by the $\gamma$-secretase complex, which has presenilin as the catalytic subunit and the generated remnant intracellular domain of MET (MET-ICD) is released into the cytosol. This fragment is extremely labile and is efficiently degraded by the proteasome (35). MET-CTF, which can escape $\gamma$-secretase cleavage, is also very labile, as it can be degraded directly via the lysosomal pathway (36) (Fig. 1A). Although
A

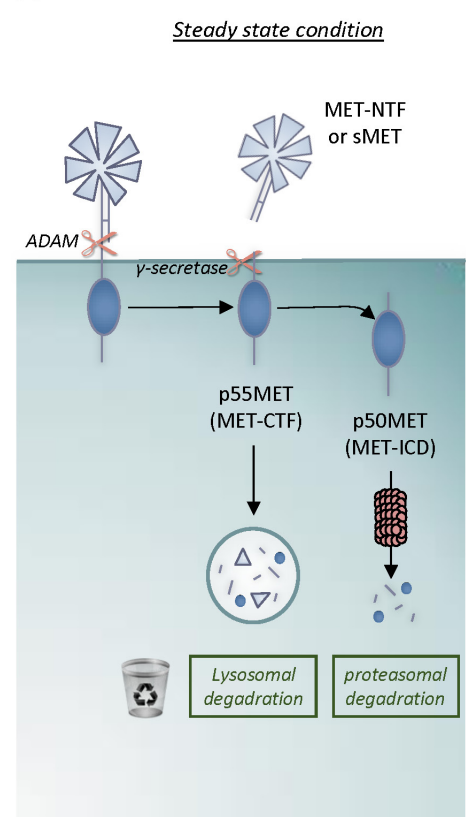

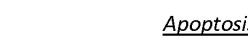

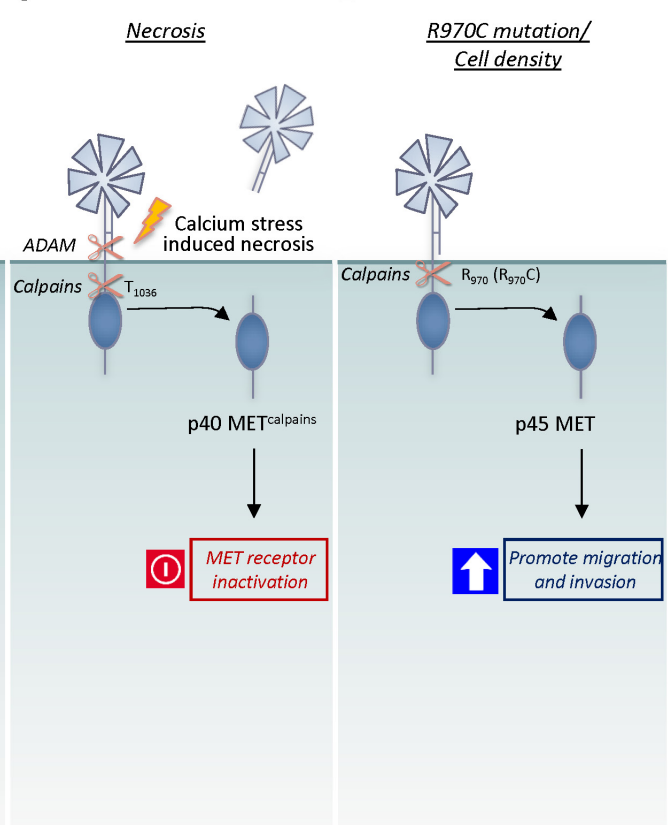

Fig. 1. The four described proteolytic cleavages of the MET receptor. (A) Presenilin-regulated intramembrane proteolysis (PS-RIP) of MET, involving sequential cleavage by ADAM metalloproteases and $\gamma$-secretase under steady-state conditions. These cleavages generate the extracellular fragment, MET-NTF (also named soluble MET, sMET) and the intracellular fragments, p55 MET (MET-CTF) and p50 MET (MET-ICD), degraded respectively by the lysosome and proteasome. This proteolytic process is involved in receptor half-life regulation. (B) Caspase cleavages of MET during apoptosis at the juxtamembrane ESVD ${ }_{1000}$ site and the C-terminal DNADDEVD ${ }_{1380}$ site, generating p40 $\mathrm{MET}^{\mathrm{T} \text { aspase }}$. This fragment can promote mitochondrial permeabilization and amplifies cell death. (C) MET cleavages during calcium-stress-induced necrosis, involving the first proteolysis by PS-RIP and a second by calcium-regulated calpains at the T1036 site, generating the intracellular p40 MET calpain fragment. (D) Calpain cleavage of MET promoted by high cell density and the R970C MET mutation. The generated intracellular p45 MET fragment can promote epithelial cell migration and invasion. 
various agents such as phorbol esters and suramin can promote MET shedding, the process occurs basally under normal conditions. With an engineered uncleavable MET whose extracellular domain is replaced with an uncleavable sequence, it has been demonstrated that PS-RIP is involved in regulating the half-life of the receptor, thus preventing its membrane accumulation (35). Consistently, pharmacological inhibition of matrix metalloproteinases induces MET accumulation and potentiates the signaling response to HGF (37). PS-RIP is thus proposed to promote basal and weak degradation of mature MET under steady-state conditions (38).

While the C-terminal fragments, MET-CTF and MET-ICD are unstable, the released N-terminal counterpart, MET-NTF, is easily detectable in the culture medium of MET-expressing cell lines and in body fluids, including blood and urine (34, 39, 40). Interestingly MET-NTF, which contains the ligand-binding domain of MET, can interact with HGF, albeit with lower affinity than the full-length receptor (34). Recombinant MET-NTF has been used as a decoy against HGF: treatment with an excess of recombinant MET-NTF inhibits HGF-induced downstream signaling pathways $(34,41)$. The physiological action of MET-NTF is still unknown.

\section{Caspase cleavages of MET: the receptor joins the dependence receptor family}

Ligand-activated MET influences cell fate through activation of anti-apoptotic signaling. Conversely, the apoptotic process influences the fate of MET in the absence of ligand: a few hours after induction of apoptosis, the MET level decreases concomitantly with the appearance of a fragment of about 40 $\mathrm{kDa}$, named p40 MET ${ }^{\text {caspase }}$. Generation of this fragment results from sequential caspase cleavages at aspartic acid 1376 or 1380 of the C-terminal tail, within a duplicated $\mathrm{DNAD}_{1376} \mathrm{DEVD}_{1380}$ consensus site (human sequence), and at aspartic acid 1002, in the ESVD 1002 site of the juxtamembrane region (Fig. 1B). The C-terminal site has been found only in mammals and is duplicated in apes and human, whereas the juxtamembrane site is highly conserved (Table 1). While juxtamembrane cleavage releases a MET receptor fragment into the cytosol, C-terminal cleavage removes only a few amino acids (14 in humans and 5 in mice). Importantly, these cleavages not only separate the extracellular ligand-binding domain from its intracellular kinase domain but also convert MET to a pro-apoptotic factor, as indicated by the fact that ectopic expression of $\mathrm{p} 40 \mathrm{MET}^{\text {caspase }}$ induces cell death concomitantly with caspase 3 activation (42). Although C-terminal cleavage removes only a few amino acids, it is required for the pro-apoptotic function of the fragment.

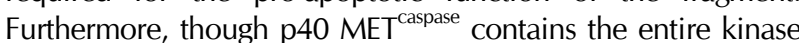
domain, the corresponding catalytic activity is not required for the pro-apoptotic function of the fragment. This suggests that the pro-apoptotic function of MET involves mechanisms distinct from the well-known intracellular signaling pathways.

Interestingly, p40 MET ${ }^{\text {caspase }}$ has been found to localize to the interface between the endoplasmic reticulum (ER) and the mitochondria, called the mitochondria-associated ER membranes (MAMs) (Duplaquet et al., submitted), suggesting an active role in mitochondria-dependent apoptosis. In fact, we have demonstrated that $440 \mathrm{MET}^{\text {caspase }}$ amplifies apoptosis by inducing mitochondrial membrane permeabilization (MMP) (43). MMP is viewed as the point of no return in the initiation of the apoptotic process, as the mitochondria release multiple pro-apoptotic factors such as cytochrome $\mathrm{C}$, involved in apoptosome formation. We have further demonstrated that p40MET promotes calcium exchange between the ER and the mitochondria, causing calcium overloading and ultimately leading to MMP (Duplaquet et al., submitted) (Fig. 1B). This original process may involve the interaction of p40 MET ${ }^{\text {caspase }}$ with the pro-apoptotic BCL2-family protein Bak, itself is recently shown to be a regulator of calcium exchange between the ER and the mitochondria (44).

Functional implications of the MET caspase cleavages were first demonstrated in cell lines where MET silencing or mutation of the C-terminal caspase site was found to prevent

Table 1. Sequences of the juxtamembrane and C-terminal caspase sites of the MET receptor in different species

\begin{tabular}{llll}
\hline \multicolumn{1}{c}{ Species } & UniProt database & \multicolumn{1}{c}{ Juxtamembrane sequence } & C-terminal sequence \\
\hline Homo sapiens & P08581 & SVSPTTEMVSNESVDYRATFPEDQ & LLSSEDNADDEVDTRPASFWETSstop \\
Pan troglodytes & Q2QLF1 & SVSPTTEMVSNESVDYRATFPEDQ & LLSSEDNADDEVDTRPASFWETSstop \\
Canis familiaris & Q75ZY9 & SVSPTTEMVSNESVDYRATFPEDQ & LLSSQDNIDGEGDTstop \\
Sus scrofas & Q2QLE0 & SVSPTTEMVSSEPVDYRATFPEDQ & LLSSQDNVDGEGDTstop \\
Rattus norvegicus & P97523 & SVSPTTEMVSNESVDYRATFPEDQ & LLPSQDNIDGEANTstop \\
Mus musculus & P16056 & SVSPTTEMVSNESVDYRATFPEDQ & LLPSQDNIDGEGNTstop \\
Xenopus laevis & Q9W650 & SISPTTEMVSSESVDYRSTVQEDP & LLSPEGNIEFSIDTstop \\
Danio rerio & Q64GK4 & SVSPTDEMVSHESVDYRTTLLEDQ & SLISSQSNLDRDstop \\
\hline
\end{tabular}

Amino-acid alignments of the juxtamembrane and C-terminal caspase sites of MET from the indicated species, extracted from UniProt data with the indicated accession number. The four amino acids of the consensus caspase sites are in blue and bold. The recruitment site of CBL (DYR) is underlined. 
cell death induced by mild stress $(43,45)$. As a next step, to assess the physiological relevance of MET caspase cleavages, we generated knock-in mice where aspartate 1374 of the C-terminal cleavage site was replaced with glutamine. In addition to preventing C-terminal cleavage, this led to the generation of a non-apoptotic form of p40 MET. Co-treatment of knock-in mice with a FAS agonist (to induce hepatocyte cell death) and a MET tyrosine kinase inhibitor (to prevent HGF-MET-dependent survival) favors resistance of the hepatocytes to apoptosis. These data demonstrate the involvement of the pro-apoptotic action of MET in regulating the survival/apoptosis balance in an organism (Duplaquet et al., submitted).

Cleavage of human MET at the C-terminal double caspase site DNADDEVD generates a short peptide of about 10 amino acids. Interestingly, ectopic expression of this peptide can directly inhibit caspase activity, which in turn contributes to the survival of hepatocytes in cell culture and animal models and reinforces the anti-apoptotic function of the receptor (46). Apparently, it is suggested that C-terminal cleavage of MET may inhibit apoptosis, in contrast to the apoptosis-amplifying effect of p40MET. Because the two caspase cleavages of MET are sequential and the C-terminal cleavage occurs before juxtamembrane cleavage (45), we propose an integrated model. According to our model, in the early steps of apoptosis, the short caspase-generated C-terminal MET fragment inhibits caspase 3 activity and protects the cell from apoptosis. At this point, the MET receptor is still activatable by its ligand and potentially able to promote survival. At a later step of apoptosis, when caspase activation reaches a higher level, MET is cleaved at the juxtamembrane site, yielding a pro-apoptotic fragment capable of promoting mitochondrial permeabilization and at this stage, MET is no longer activatable by its ligand (47).

The view that MET exerts opposite responses in the presence and absence of its ligand is strengthened by the observation that MET tyrosine phosphorylation prevents caspase cleavage of MET. The ESVD ${ }_{1002}$ juxtamembrane cleavage site is followed by the phosphorylated $\mathrm{Y} 1003$ residue, known to be crucial for CBL recruitment. Residue D1002 belongs to the DYR site recognized by $C B L \quad\left(E S V D_{1002} Y_{1003} R\right)$ (48). Phosphorylation of the tyrosine residue prevents p40 MET cleavage by caspases (49). These data demonstrate that activation of MET by its ligand prevents deployment of its apoptotic capacity. The $\mathrm{N}$-terminal counterpart of p40 MET, an approximately 100-kDa membrane-anchored fragment, can still bind HGF and may act as a decoy against HGF, thus also contributing to promoting cell death (50).

The pro-apoptotic function mediated by the MET receptor in the absence of its ligand (in contrast with its anti-apoptotic function in response to HGF/SF) is the hallmark of the dependence receptor family. Although a receptor is usually considered inactive until bound by its ligand, some receptors are known to induce cell death in the absence of ligand. Cells expressing such receptors thus become dependent on the receptor-ligand for survival (51). This dependence phenomenon has been well described for several membrane receptors, including the TRKC, TRKA, KIT, RET, EPHA4, ALK, and IGF-1R receptor tyrosine kinases (52). For instance, TRKC, like MET, undergoes two caspase cleavages releasing a TRKC "killer fragment" (TRKC-KF), which localizes to the mitochondria and favors Bax activation via interaction with COBRA1, and promotes MMP, a mechanism reminiscent of p40 METinduced apoptosis (53). TRC-KF also localizes to the nucleus, where it interacts with HEY1, a transcription factor involved in negative regulation of mouse double minute 2 (MDM2), an E3 ubiquitin ligase, which regulates p53 stability. Thus, TRKC-KF can also promote apoptosis via p53 stabilization (54). Interestingly, p40 MET can also localize to the nucleus, where one might hypothesize its interaction with transcriptional regulators.

\section{Calcium-dependent calpain cleavages of MET: receptor inactivation during necrosis}

Although necrosis was long viewed as accidental cell death, it is currently regarded as a programmed cell death mechanism, as certain pharmacological inhibitors and gene deletions can protect cells from this demise. Necrosis is a caspase- and ATP-independent cell death mechanism featuring early plasma membrane and organelle disruption. Many pathways can lead to cell necrosis, including calcium overload. This type of cell stress has been extensively described in the nervous system, where an increase in intracellular calcium results in neuronal injury and neurodegenerative disease. In many other cell types, calcium ionophores such as ionomycin can induce rapid necrosis. An increase in intracellular calcium triggers the activation of several proteases, including calpains and cathepsins (55-57).

We have demonstrated that calcium-stress-induced necrosis causes a substantial decrease in MET within minutes following treatment with a calcium ionophore. Consistently with this decrease, HGF stimulation can no longer trigger MET phosphorylation or activation of downstream signaling pathways. The observed MET degradation might thus prevent the cell protection resulting from ligand-dependent activation of MET. Rapid MET degradation is associated with detection of an extracellular and an intracellular fragment. The extracellular fragment (about $90 \mathrm{kDa}$ ) is generated as a consequence of MET cleavage by ADAM-family membrane metalloproteases, causing MET receptor shedding through a mechanism similar to the above-described PS-RIP (section 1). Consequently, an extracellular fragment containing the ligand-binding domain of MET is released into the extracellular space. This initial extracellular cleavage is followed by intracellular cleavage by $\gamma$-secretase, generating the labile MET-CTF fragment (about 50 kDa) (Fig. 1C) (59). Necrosis also induces the generation of a stable intracellular fragment of about $40 \mathrm{kDa}$. This fragment results from receptor cleavage by the calcium-dependent 
calpain proteases activated during necrosis. Calpains are calcium-dependent proteases capable of cleaving multiple substrates and involved in regulating various cell processes, including migration, autophagy, apoptosis, and necrosis. Interestingly, the effector role of calpains during necrosis is reminiscent of the function of caspases during apoptosis. Caspases are directly involved in apoptotic morphological changes, while calpains cleave the cytoskeletal proteins such as spectrin and tubulin, thus favoring necrotic dismantling of cell structure (59-61). Calpain cleavage of MET occurs at residue T1036 in the juxtamembrane domain and generates a fragment close to the $\mathrm{p} 40 \mathrm{MET}^{\text {caspase }}$ fragment generated during apoptosis since only 34 residues separate it from the caspase site (D1002). Therefore, the fragment of about $40 \mathrm{kDa}$ generated by calpains is called p40 MET $T^{\text {calpain }}$. Despite the similarities, p40 $\mathrm{MET}^{\text {calpain }}$ conserves its C-terminal tail, as unlike p40 $\mathrm{MET}^{\text {caspase }}$, it does not undergo C-terminal cleavage. Furthermore, in contrast to $\mathrm{p} 40 \mathrm{MET}^{\text {caspase }}$, the reconstituted p40 MET ${ }^{\text {calpain }}$ fragment does not display apoptotic activity (Fig. 1C).

At first glance, the mechanism of MET degradation appears similar during apoptosis and calcium-stress-induced necrosis: in both the cases, it involves the generation of an intracellular fragment of about $40 \mathrm{kDa}$. In both the processes, the extracellular ligand-binding domain of MET is separated from the intracellular kinase domain, thus preventing survival. However, calcium-stress-induced necrosis triggers a more complex proteolytic process involving cleavage by membrane metalloproteases. The cleavages occurring during necrosis are quicker than caspase cleavages, leading to MET degradation within minutes of stress induction. In addition, while p40MET ${ }^{\text {caspase }}$ somewhat resembles $\mathrm{p} 40 \mathrm{MET}^{\text {calpain }}$, the two fragments appear functionally different: the former can favor cell death while the latter is unable to promote such a response.

\section{The R970C mutation and high cell density favor calpain-dependent cleavages of MET}

As reported previously, most of the proteolytic cleavages affecting MET occur in the juxtamembrane domain. In lung cancer, interestingly, somatic mutations of MET also affect the juxtamembrane domain. The first group of mutations impairs the splice acceptor and donor sites of exon 14, leading to exon skipping and deletion of a large part of the juxtamembrane domain. These mutations have been found in about $3 \%$ of NSCLCs and include various point mutations and deletions, all targeting the splice sites. Deletion of the juxtamembrane domain favors receptor activation by its ligand, as the deleted domain contains several negative regulatory sites (62). The second group of mutations affecting the juxtamembrane domain comprises point mutations, which cause amino acid substitutions within the domain, notably the R970C, P991S, and T992I substitutions (respectively R988C, P1009S, and $\mathrm{T} 1010 \mathrm{l}$ in the long isoform of MET). The R970C variant, for instance, is found in about $1 \%$ of patients (27). In contrast to mutations affecting splice sites or the kinase domain, it is unknown how these amino acid substitutions within the juxtamembrane domain functionally affect MET. While studies have demonstrated that they favor the growth of experimental tumors, they do not cause MET kinase activation (63-65). In addition, although these mutations were initially identified in lung tumors, recent studies have shown that they can be germline variants corresponding to polymorphisms (27, 65-67). Yet in the mouse strain SWRJ, the R968C MET variant (corresponding to the human R970C variant) favors the development of lung tumors, suggesting that it modifies MET activity through an unknown mechanism (68).

In stably transfected epithelial cells expressing a mutated form of MET, the juxtamembrane mutations R970C, P991S, and T992I have been found not to modify the known caspaseor presenilin-dependent intramembrane proteolyzes. However, when overexpressed, the R970C variant was found to cause generation of an as yet undescribed 45-kDa fragment (named p45 MET). This fragment was also found in confluent $\mathrm{NCl}-\mathrm{H} 1437$ lung cancer cells carrying the R970C mutation, and to a lesser extent in confluent cells expressing WT MET, suggesting that the R970C mutation is not required but favors this cleavage. Calpain inhibitors were found to prevent p45 MET generation, suggesting the involvement of this protease family. The apparent molecular size of the p45 MET fragment is consistent with a juxtamembrane cleavage occurring near position 970, but it is difficult to predict the exact cleavage site, since no consensus calpain cleavage sites have been described (Fig. 1D). In contrast to cleavages occurring in the course of apoptosis or necrosis, which is accompanied by a decrease in full-length MET, calpain cleavage at the R970 site does not alter the level of full-length MET, thus suggesting that this proteolytic cleavage is not involved in downregulating the level of full-length receptor. Interestingly, ectopic expression of the reconstituted p45 MET in epithelial cell lines favors cell scattering and invasion, induced or not by HGF, indicating an active role of this fragment (69). As the R970C mutation promotes p45 MET fragment generation, this somatic mutation might be viewed as a novel type of activating mutation, creating an active fragment that promotes cell motility.

The molecular mechanism through which p45 MET promotes cell invasion remains elusive. Full-length MET promotes intracellular signaling through phosphorylation of its tyrosines involved notably in recruiting intracellular adaptors. In contrast, endogenous p45 MET does not display tyrosine phosphorylation, even following HGF/SF treatment, thus suggesting that it does not promote epithelial cell scattering through the same mechanisms as phosphorylated full-length MET.

Labile versus stable MET fragments

As presented above, MET undergoes distinct proteolytic cleavages leading to the generation of various intracellular 
fragments defined as, the labile and stable ones. The labile fragments are p55 MET (MET-NTF) and p50 MET (MET-ICD), generated by the ADAM metalloproteases and $\gamma$-secretase during PS-RIP. Accordingly, these labile fragments are related to receptor degradation. The stable fragments are p45 MET, generated by calpain proteases, and p40 MET ${ }^{\text {caspase }}$ and p40 MET $^{\text {calpain }}$ generated respectively by caspases and calciumregulated calpain. MET fragment stability could thus be related to a short, 14-aa sequence (956-KKRKQIKDLGSELVR-970) of the juxtamembrane domain, present in p50 MET and absent in p45 MET (Fig. 2A and 2B).

At first glance, it seems that the labile fragments are associated with MET degradation since PS-RIP is involved in regulating the MET half-life under steady-state conditions. In contrast, the stable fragments might be associated with regulating biological responses, as demonstrated for the pro-invasive p45 MET fragment and the pro-apoptotic p40 MET $^{\text {caspase }}$ fragment. The situation might be less clear-cut, as some intracellular domain fragments (ICD fragments) generated by PS-RIP can, in fact, translocate into the nucleus to regulate transcription, like ICD of ERBB4 (70). Interestingly, intracellular C-terminal fragments of MET fused to the Gal-4 DNA-binding domain have been shown to display transactivation properties, which is inhibited by YAP silencing (71). In addition, a recent study has mapped a nuclear localization signal to the juxtamembrane region of MET $(\mathrm{H} 1068-\mathrm{H} 1079)$ and suggests that a MET fragment might act as a transcription regulator via a mechanism involving the transcriptional regulator YAP (72). The observed instability of p50 MET (MET-ICD) should rule out its involvement in transcriptional regulation unless particular cellular conditions favor its stability. Inversely, the p40 MET calpain fragment produced during necrosis is stable, although no biological activity has been associated with it. In particular, no cytotoxicity has been associated with its generation, in contrast to what might be expected of a fragment produced during necrosis.

\section{MET CLEAVAGES AND CANCER}

Because MET is a well-known oncogenic protein frequently activated in cancer, it has been proposed that MET cleavages might play a role in cell transformation. In this regard, the proteolytic cleavages of MET can be categorized into two groups: (i) cleavages that might promote cell transformation and (ii) cleavages that might prevent cell transformation, notably through MET inactivation. There is currently only limited experimental evidence to demonstrate an understanding of the involvement of MET cleavages in tumorigenesis; apparently, crucial in vivo studies are still required. On the other hand, proteolytic cleavages of MET have been exploited in developing potential therapeutic tools or strategies for inhibiting cell transformation and tumorigenesis.

\section{Is calpain proteolysis of MET the missing link between MET cleavage and cancer?}

Only one proteolytic cleavage of MET produces an intracellular fragment capable of triggering biological responses
A

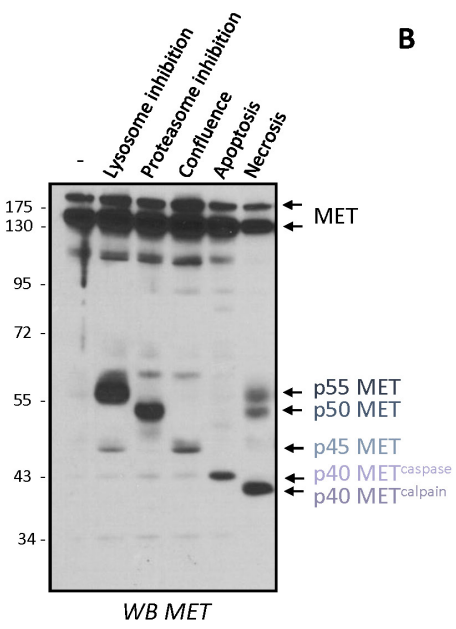

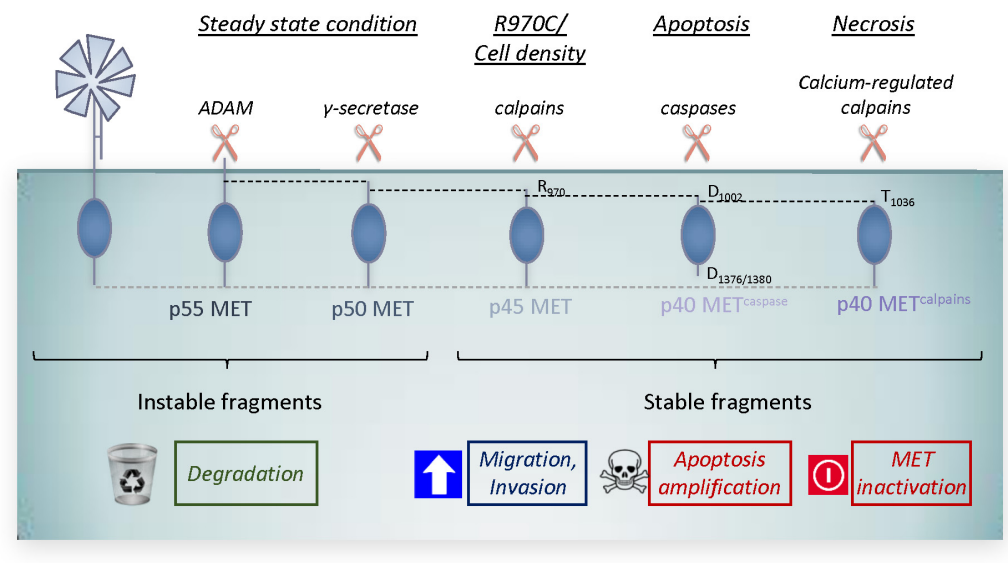

Fig. 2. Unstable and stable intracellular MET fragments produced by proteolytic cleavages. (A) MCF-10A cells were subjected to the following treatments: incubation with a lysosome inhibitor $(5 \mathrm{nM}$ bafilomycin for $5 \mathrm{~h}$ ); incubation with a proteasome inhibitor (10 $\mu \mathrm{M}$ lactacystin for $5 \mathrm{~h}$ ); culturing to high density (confluence); incubation with an apoptosis inducer (1 $\mu \mathrm{M}$ staurosporine for $6 \mathrm{~h}$ ); and induction of necrosis (with $1 \mu \mathrm{M}$ ionomycin for $1 \mathrm{~h}$ ). Cell lysates were analyzed by western blotting with an antibody directed against the kinase domain of human MET. Arrows indicate the positions of MET and of the different MET fragments, p55 MET, p50 MET, p45 MET, p40 MET $\mathrm{Maspase}^{\text {, and }} \mathrm{p} 40 \mathrm{MET}^{\text {calpain }}$ (see (58) for detailed experimental procedures). (B) Schematic representation of the known unstable and stable intracellular MET fragments generated by proteolytic cleavages. 
consistent with cell transformation: calpain cleavage at residue R970 in the juxtamembrane domain which results in the generation of p45 MET. Ectopic expression of p45 MET promotes the motility and invasion by epithelial cells, the two effects known to favor tumorigenesis. In addition, p45 MET generation is favored by R970C mutations found at a low rate in lung cancers, suggesting a causative link between the proteolytic cleavage and cancer. Finally, high cell density favors p45 MET generation, consistently with an observed increase in calpain activity under such conditions (69). Altogether, this suggests that high cell density and R970C mutation could promote cell migration and invasion through MET cleavages, thus favoring the escape of tumor cells from a primary tumor. However, crucial experiments in animal models like a demonstration that ectopic expression of the p45 MET fragment or expression of the MET R970C variant favors tumor growth, or that patients harboring R970C produce more p45 MET fragments are necessitated.

\section{How can caspase cleavages convert MET from an oncogene to a tumor suppressor?}

Dependence receptors constitute what is now known as a large family of receptors sharing the same functional feature: the ability to promote survival in the presence of ligand and apoptosis in its absence. Some RTKs such as MET (42), TRKC (73), RET (74), and KIT (75) are dependence receptors. Interestingly, all these receptors were initially known as oncoproteins and were found capable of inducing tumorigenesis when overactivated by aberrant ligand stimulation, mutations, overexpression, or even chromosomal translocation. Yet their ability to induce cell death in the absence of ligand reflects an opposite action as tumor suppressors. For instance, TRKC downregulation, notably through promoter methylation, has been demonstrated in colon cancer. Furthermore, re-established TRKC expression in colorectal cancer cell lines is associated with tumor cell death and inhibition of tumor growth $(76,77)$.

Like most RTKs, MET is known mainly as an oncoprotein. Nevertheless, as a member of the dependence receptor family, it might also be expected to act as a tumor suppressor. High levels of some members of this family, such as TRKC, RET, and KIT, have been associated with a good prognosis; however, this is not the case with MET and there exist no reported data on molecular alterations leading to MET downregulation in cancer. Several studies show that MET overproduction occurs in cancers and is associated with poor prognosis $(19,78)$. Nevertheless, it is uncertain whether the tumorigenic potential of MET overactivation is solely due to kinase domain activity or if inhibition of the MET apoptotic capacity might also have an impact, as MET activation leads to inhibition of MET cleavage by caspases and of its ability to amplify apoptosis. We can thus propose that, although activation of MET pro-survival signaling is known to contribute to its oncogenic activity, loss of MET pro-apoptotic capacity might also be crucial in unleashing the tumorigenic activity of this receptor.

Apparently, the COSMIC database (Catalogue of Somatic Mutations in Cancer) now lists ten mutations affecting MET caspase sites in various cancers, such as D1002N in colorectal cancer or D1380N in ovarian cancer. As each mutation has been recorded only once, it would seem that such mutations occur at a low rate. However, recently it has been shown that about $3 \%$ of NSCLCs display mutations affecting the splice site of MET exon 14, causing deletion of the juxtamembrane region, where a caspase cleavage site has been found (62). Thus, MET $\Delta$ exon14 mutants are virtually uncleavable by caspases and might contribute to tumorigenesis by promoting a stronger pro-survival response.

In vivo evidence is required to assess the pro-apoptotic role of MET in tumorigenesis. It could be interesting, to see if knock-in mice mutated at the MET caspase site develop spontaneous tumors with aging or whether their breeding with mice prone to spontaneous tumorigenesis increases penetrance or the number of tumors.

\section{Using soluble MET for therapy and diagnosis}

As described above, PS-RIP of MET generates an extracellular $\mathrm{N}$-terminal fragment (MET-NTF or sMET). This fragment, comprising the ligand-binding domain, can bind to HGF and act as a decoy (34), as confirmed by the observation that an excess of engineered recombinant sMET can interfere with both HGF binding to MET and MET homodimerization. In addition, sMET can inhibit tumor cell proliferation and survival in a variety of human xenograft models (41), acting as a potential therapeutic tool through its decoy activity.

Another therapeutic strategy might be forced induction of MET PS-RIP, for instance in cancer cells overexpressing the receptor. This could lead both to a decreased level of MET and to the generation of the decoy SMET. Although no natural stimulation has been shown to induce PS-RIP, the monoclonal antibody DN30 developed by P. Comoglio's team promotes both $\operatorname{ADAM}(33,79)$ and $\gamma$-secretase cleavages (35). This antibody, directed against the MET extracellular domain, can trigger strong degradation of the receptor, concomitantly with the generation of SMET in the extracellular medium and its unstable counterpart fragments inside the cell $(35,79)$. DN30 antibody has been shown to reduce tumor growth in animals xenografted with various tumor cells displaying constitutive MET activation, notably due to MET gene amplification. Extensive studies have been carried out with an aim to improve DN30 efficacy. Extensive research has notably focused on developing a monovalent version to prevent MET dimerization (80), increasing DN30 stability by molecular and chemical modifications $(81,82)$, and enhancing its delivery through gene (antibody coding sequence) transfer (83). Another elegant approach has been to combine the DN30 antibody with an altered SMET decoy unable to bind DN30 because of a point mutation in its DN30-binding domain. These molecules have been observed to cooperate in 
restraining cancer cell invasion and experimental tumor growth (84). The DN30 antibody has also been coupled with positron emitters such as ${ }^{89} \mathrm{Zr}$ for positron emission tomography (PET). MET-expressing xenografted tumors as small as about $10 \mathrm{mg}$ were readily visualized by immuno-PET using ${ }^{89} \mathrm{Zr}$-DN30 (85).

As MET shedding through PS-RIP is constitutive, sMET generation seems proportional to the level of full-length MET in cells $(34,39)$. Also, because sMET is uncoupled from the cell plasma membrane, it is readily detectable in body fluids $(34,39,40)$. It has therefore been proposed that MET measured in blood sample might be used as a diagnostic tool to assume the level of full-length MET in a tumor. In mice xenografted with human tumor cells, a direct correlation has been found between tumor volume and the concentration of human SMET in the blood (86). The concentration of soluble MET has also been measured in sera from one French and two Chinese cohorts of patients with non-small-cell lung cancer. In the two Chinese cohorts, the sMET level was found to increase with the level of full-length MET within the tumor, but in the French cohort, no correlation was found. It is worth noting that the patients of both the Chinese cohorts displayed a high EGFR mutation rate, as is usually found in the Asiatic population. In the study by $\mathrm{Fu}$ et al., the selected patients harbored EGFR mutations and were treated with an EGFR inhibitor (87). In the study by Lv et al., about a quarter of the NSCLC patients displayed EGFR mutations and received an EGFR inhibitor (88). In the patients of the French cohort, EGFR mutations were absent. Importantly, treatment is well known to influence the molecular profile of lung tumors. For instance, resistance to EGFR-targeting therapies has been linked to amplification of the MET gene, leading to its massive expression in about $20 \%$ of patients (89). Thus, the high level of SMET in the Chinese cohort could be due to MET gene amplification. Furthermore, in the study by Fu et al (87), the tumor diameter was large in the patients showing the highest SMET levels, thus suggesting that large tumors may generate more sMET. Lastly, measuring sMET in the urine allows differentiation between patients with and without bladder cancer. Urinary SMET might thus be used as a bladder cancer biomarker (40).

\section{CONCLUSION}

The signal-transducing activity of RTKs, including MET, relies on stimulation by the ligand and activation of downstream signaling pathways. As it is clearly related to the tyrosine kinase domain, RTK cleavages are often considered anecdotic, and their possible biological action is neglected. However, for receptors that do not display kinase activity, signaling through proteolytic cleavage is the rule. In Notch signaling, for example, secretase cleavages allow generation of an intracellular fragment capable of transducing the information through its translocation into the nucleus, where it can regulate transcriptional responses (90). In the case of MET, the role of cleavages first appeared restricted to receptor downregulation. The distribution of roles thus appeared to be: tyrosine kinase activity for signal transduction and proteolysis for receptor degradation. Recent findings, however, have highlighted a more complex situation: some MET fragments are active and can regulate invasion or the survival/apoptosis balance. Thus, MET cleavages seem more important than initially suspected, and may help to fully explain the wide range of biological responses regulated by MET. Interestingly, all the MET proteolytic cleavages described to date occur independently of ligand stimulation. This suggests a different distribution of roles, with tyrosine kinase activity regulating the ligand-dependent responses and proteolytic cleavages regulating the ligand-independent responses. Besides the possibility of discovering novel proteolytic cleavages, the current challenge is to completely understand the biological consequences of the MET cleavages already described. For instance, an issue likely to be important in pathological situations such as cancer is: how do MET intracellular fragments, including p45 MET, promote epithelial cell invasion? It is intriguing to propose that such fragments might translocate into the nucleus to regulate transcriptional responses, as in the case of prototypical cleaved receptors such as Notch.

\section{ACKNOWLEDGEMENTS}

This work was supported by the CNRS, the Institut Pasteur de Lille, and INSERM, and by grants from the "Cancéropôle Nord-Ouest", the "Agence Nationale de la Recherche; Metropolis project", the "Ligue Contre le Cancer, Comité Nord", the "Institut National du Cancer" and "Ecole Doctorale Biologie Santé".

\section{CONFLICTS OF INTEREST}

The authors have no conflicting interests.

\section{REFERENCES}

1. Birchmeier C, Birchmeier W, Gherardi E and Vande Woude GF (2003) Met, metastasis, motility and more. Nat Rev Mol Cell Biol 4, 915-925

2. Bladt F, Riethmacher D, Isenmann S, Aguzzi A and Birchmeier C (1995) Essential role for the c-met receptor in the migration of myogenic precursor cells into the limb bud. Nature 376, 768-771

3. Schmidt C, Bladt F, Goedecke S et al (1995) Scatter factor/hepatocyte growth factor is essential for liver development. Nature 373, 699-702

4. Uehara Y, Minowa O, Mori $C$ et al (1995) Placental defect and embryonic lethality in mice lacking hepatocyte growth factor/scatter factor. Nature 373, 702-705

5. Maina F, Hilton MC, Ponzetto C, Davies AM and Klein R 
(1997) Met receptor signaling is required for sensory nerve development and HGF promotes axonal growth and survival of sensory neurons. Genes Dev 11, 3341-3350

6. Chmielowiec J, Borowiak M, Morkel M et al (2007) c-Met is essential for wound healing in the skin. J Cell Biol 177, 151-162

7. Borowiak M, Garratt AN, Wustefeld T, Strehle $M$, Trautwein C and Birchmeier C (2004) Met provides essential signals for liver regeneration. Proc Natl Acad Sci U S A 101, 10608-10613

8. Huh CG, Factor VM, Sanchez A, Uchida K, Conner EA and Thorgeirsson SS (2004) Hepatocyte growth factor/c-met signaling pathway is required for efficient liver regeneration and repair. Proc Natl Acad Sci U S A $101,4477-4482$

9. Schiering N, Knapp S, Marconi M et al (2003) Crystal structure of the tyrosine kinase domain of the hepatocyte growth factor receptor c-Met and its complex with the microbial alkaloid K-252a. Proc Natl Acad Sci U S A 100, 12654-12659

10. Longati $P$, Bardelli A, Ponzetto $C$, Naldini L and Comoglio PM (1994) Tyrosines1234-1235 are critical for activation of the tyrosine kinase encoded by the MET protooncogene (HGF receptor). Oncogene 9, 49-57

11. Ponzetto C, Bardelli A, Zhen Z et al (1994) A multifunctional docking site mediates signaling and transformation by the hepatocyte growth factor/scatter factor receptor family. Cell 77, 261-271

12. Maina F, Casagranda F, Audero E et al (1996) Uncoupling of Grb2 from the Met receptor in vivo reveals complex roles in muscle development. Cell 87, 531-542

13. Peschard P, Fournier TM, Lamorte L et al (2001) Mutation of the c-Cbl TKB domain binding site on the Met receptor tyrosine kinase converts it into a transforming protein. Mol Cell 8, 995-1004

14. Hashigasako A, Machide M, Nakamura T and Matsumoto K (2004) Bi-directional regulation of Ser-985 phosphorylation of c-met via protein kinase $\mathrm{C}$ and protein phosphatase $2 \mathrm{~A}$ involves c-Met activation and cellular responsiveness to hepatocyte growth factor. J Biol Chem 279, 26445-26452

15. Duplaquet L, Kherrouche Z, Baldacci S et al (2018) The multiple paths towards MET receptor addiction in cancer. Oncogene 37, 3200-3215

16. Olivero M, Rizzo M, Madeddu R et al (1996) Overexpression and activation of hepatocyte growth factor/scatter factor in human non-small-cell lung carcinomas. $\mathrm{Br}$ J Cancer 74, 1862-1868

17. Ichimura E, Maeshima A, Nakajima $T$ and Nakamura $T$ (1996) Expression of c-met/HGF receptor in human non-small cell lung carcinomas in vitro and in vivo and its prognostic significance. Jpn J Cancer Res 87, 1063-1069

18. Wislez M, Rabbe N and Marchal J (2003) Hepatocyte growth factor production by neutrophils infiltrating bronchioloalveolar subtype pulmonary adenocarcinoma: role in tumor progression and death. Cancer Res 63, 1405-1412

19. Park S, Choi YL, Sung CO et al (2012) High MET copy number and MET overexpression: poor outcome in non-small cell lung cancer patients. Histol Histopathol 27, 197-207
20. Koochekpour S, Jeffers M, Rulong S, et al (1997) Met and hepatocyte growth factor/scatter factor expression in human gliomas. Cancer Res 57, 5391-5398

21. Ferracini R, Di Renzo MF, Scotlandi K et al (1995) The Met/HGF receptor is over-expressed in human osteosarcomas and is activated by either a paracrine or an autocrine circuit. Oncogene 10, 739-749

22. Tuck AB, Park M, Sterns EE, Boag A and Elliott BE (1996) Coexpression of hepatocyte growth factor and receptor (Met) in human breast carcinoma. Am J Pathol 148, 225-232

23. Ponzetto C, Giordano S, Peverali F et al (1991) c-met is amplified but not mutated in a cell line with an activated met tyrosine kinase. Oncogene 6, 553-559

24. Watermann I, Schmitt B and Stellmacher F (2015) Improved diagnostics targeting C-MET in non-small cell lung cancer: expression, amplification and activation? Diagn Pathol 10, 130

25. Yang Y, Wu N, Shen J et al (2016) MET overexpression and amplification define a distinct molecular subgroup for targeted therapies in gastric cancer. Gastric Cancer 19, 778-788

26. Sattler M, Reddy MM, Hasina R, Gangadhar T and Salgia $R$ (2011) The role of the c-Met pathway in lung cancer and the potential for targeted therapy. Ther Adv Med Oncol 3, 171-184

27. Krishnaswamy S, Kanteti R, Duke-Cohan JS et al (2009) Ethnic differences and functional analysis of MET mutations in lung cancer. Clin Cancer Res 15, 5714-5723

28. Baldacci S, Mazieres J, Tomasini P et al (2017) Outcome of EGFR-mutated NSCLC patients with MET-driven resistance to EGFR tyrosine kinase inhibitors. Oncotarget 8, 105103-105114

29. Prat M, Crepaldi T, Gandino L, Giordano S, Longati P and Comoglio P (1991) C-terminal truncated forms of Met, the hepatocyte growth factor receptor. Mol Cell Biol 11, 5954-5962

30. Galvani AP, Cristiani C, Carpinelli P, Landonio A and Bertolero F (1995) Suramin modulates cellular levels of hepatocyte growth factor receptor by inducing shedding of a soluble form. Biochem Pharmacol 50, 959-966

31. Jeffers $M$, Taylor GA, Weidner KM, Omura $S$ and Vande-Woude GF (1997) Degradation of the Met tyrosine kinase receptor by the ubiquitin-proteasome pathway. Mol Cell Biol 17, 799-808

32. Kopitz C, Gerg M, Bandapalli OR et al (2007) Tissue inhibitor of metalloproteinases-1 promotes liver metastasis by induction of hepatocyte growth factor signaling. Cancer Res 67, 8615-8623

33. Schelter F, Kobuch J, Moss ML et al (2010) A disintegrin and metalloproteinase-10 (ADAM-10) mediates DN30 antibody-induced shedding of the met surface receptor. J Biol Chem 285, 26335-26340

34. Wajih N, Walter J and Sane DC (2002) Vascular origin of a soluble truncated form of the hepatocyte growth factor receptor (c-met). Circ Res 90, 46-52

35. Foveau B, Ancot F and Leroy C (2009) Downregulation of the Met Receptor Tyrosine Kinase by Presenilindependent Regulated Intramembrane Proteolysis. Mol Biol Cell 20, 2494-2506 
36. Ancot F, Leroy C, Muharram G et al (2012) SheddingGenerated Met Receptor Fragments can be Routed to Either the Proteasomal or the Lysosomal Degradation Pathway. Traffic 13, 1261-1272

37. Miller MA, Meyer AS and Beste MT (2013) ADAM-10 and -17 regulate endometriotic cell migration via concerted ligand and receptor shedding feedback on kinase signaling. Proc Natl Acad Sci U S A 110, E2074-2083

38. Trusolino L, Bertotti A and Comoglio PM (2010) MET signalling: principles and functions in development, organ regeneration and cancer. Nat Rev Mol Cell Biol 11, 834-848

39. Copin MC, Lesaffre M, Berbon M et al (2016) High-MET status in non-small cell lung tumors correlates with receptor phosphorylation but not with the serum level of soluble form. Lung Cancer 101, 59-67

40. McNeil BK, Sorbellini M and Grubb RL 3rd et al (2014) Preliminary evaluation of urinary soluble Met as a biomarker for urothelial carcinoma of the bladder. J Transl Med 12, 199

41. Michieli P, Mazzone M, Basilico C et al (2004) Targeting the tumor and its microenvironment by a dual-function decoy Met receptor. Cancer Cell 6, 61-73

42. Tulasne D, Deheuninck J, Lourenco FC et al (2004) Proapoptotic function of the MET tyrosine kinase receptor through caspase cleavage. Mol Cell Biol 24, 10328-10339

43. Lefebvre J, Muharram G, Leroy C et al (2013) Caspasegenerated fragment of the Met receptor favors apoptosis via the intrinsic pathway independently of its tyrosine kinase activity. Cell Death Dis 4, e871

44. Mebratu YA, Leyva-Baca I and Wathelet MG (2017) Bik reduces hyperplastic cells by increasing Bak and activating DAPk1 to juxtapose ER and mitochondria. Nat Commun 8, 803

45. Foveau B, Leroy C, Ancot F et al (2007) Amplification of apoptosis through sequential caspase cleavage of the MET tyrosine kinase receptor. Cell Death Differ 14, 752-764

46. Ma J, Zou C and Guo L (2013) A novel death defying domain in met entraps the active site of caspase- 3 and blocks apoptosis in hepatocytes. Hepatology 59, 20102021

47. Furlan A and Tulasne D (2013) How does met regulate the survival/apoptosis balance? Hepatology 60, 1108-1109

48. Peschard $P$, Ishiyama $N$, Lin $T$, Lipkowitz $S$ and Park $M$ (2004) A conserved DpYR motif in the juxtamembrane domain of the Met receptor family forms an atypical c-Cbl/Cbl-b tyrosine kinase binding domain binding site required for suppression of oncogenic activation. J Biol Chem 279, 29565-29571

49. Deheuninck J, Goormachtigh G and Foveau B (2009) Phosphorylation of the MET receptor on juxtamembrane tyrosine residue 1001 inhibits its caspase-dependent cleavage. Cell Signal 21, 1455-1463

50. Deheuninck J, Foveau B, Goormachtigh G et al (2008) Caspase cleavage of the MET receptor generates an HGF interfering fragment. Biochem Biophys Res Commun 367, 573-577

51. Bredesen DE, Mehlen P and Rabizadeh S (2005) Receptors that mediate cellular dependence. Cell Death Differ 12, 1031-1043
52. Negulescu AM and Mehlen P (2018) Dependence receptors - the dark side awakens. FEBS J 285, 3909-3924

53. Ichim G, Genevois AL, Menard M et al (2013) The Dependence Receptor TrkC Triggers MitochondriaDependent Apoptosis upon Cobra-1 Recruitment. Mol Cell 51, 632-646

54. Menard M, Costechareyre C, Ichim G et al (2018) Hey1and p53-dependent TrkC proapoptotic activity controls neuroblastoma growth. PLoS Biol 16, e2002912

55. Halestrap AP (2010) A pore way to die: the role of mitochondria in reperfusion injury and cardioprotection. Biochem Soc Trans 38, 841-860

56. Francis RJ, Kotecha $S$ and Hallett MB (2013) $\mathrm{Ca}^{2+}$ activation of cytosolic calpain induces the transition from apoptosis to necrosis in neutrophils with externalized phosphatidylserine. J Leukoc Biol 93, 95-100

57. Yun B, Lee H, Ghosh $M$ et al (2014) Serine hydrolase inhibitors block necrotic cell death by preventing calcium overload of the mitochondria and permeability transition pore formation. J Biol Chem 289, 1491-1504

58. Montagne R, Berbon M, Doublet L et al (2015) Necrosisand apoptosis-related Met cleavages have divergent functional consequences. Cell Death Dis 6, e1769

59. Billger M, Wallin M and Karlsson JO (1988) Proteolysis of tubulin and microtubule-associated proteins 1 and 2 by calpain I and II. Difference in sensitivity of assembled and disassembled microtubules. Cell Calcium 9, 33-44

60. Czogalla A and Sikorski AF (2005) Spectrin and calpain: a 'target' and a 'sniper' in the pathology of neuronal cells. Cell Mol Life Sci 62, 1913-1924

61. Kelly BL, Vassar R and Ferreira A (2005) Beta-amyloidinduced dynamin 1 depletion in hippocampal neurons. A potential mechanism for early cognitive decline in Alzheimer disease. J Biol Chem 280, 31746-31753

62. Cortot AB, Kherrouche Z, Descarpentries C et al (2017) Exon 14 deleted MET receptor as a new biomarker and target in cancers. J Natl Cancer Inst 109

63. Lee JH, Han SU, Cho $\mathrm{H}$ et al (2009) A novel germ line juxtamembrane Met mutation in human gastric cancer. Oncogene 19, 4947-4953

64. Ma PC, Kijima T, Maulik G et al (2003) c-MET mutational analysis in small cell lung cancer: novel juxtamembrane domain mutations regulating cytoskeletal functions. Cancer Res 63, 6272-6281

65. Tyner JW, Fletcher LB, Wang EQ et al (2010) MET receptor sequence variants $\mathrm{R} 970 \mathrm{C}$ and T992l lack transforming capacity. Cancer Res 70, 6233-6237

66. Shieh JM, Tang YA, Yang TH et al (2013) Lack of association of C-Met-N375S sequence variant with lung cancer susceptibility and prognosis. Int J Med Sci 10, 988-994

67. Boland JM, Jang JS, Li J et al (2013) MET and EGFR mutations identified in ALK-rearranged pulmonary adenocarcinoma: molecular analysis of 25 ALK-positive cases. J Thorac Oncol 8, 574-581

68. Zaffaroni D, Spinola M, Galvan A et al (2005) Met proto-oncogene juxtamembrane rare variations in mouse and humans: differential effects of Arg and Cys alleles on mouse lung tumorigenesis. Oncogene 24, 1084-1090

69. Montagne R, Baranzelli A, Muharram G et al (2017) MET 
receptor variant R970C favors calpain-dependent generation of a fragment promoting epithelial cell scattering. Oncotarget 8, 11268-11283

70. Merilahti JAM and Elenius K (2019) Gamma-secretasedependent signaling of receptor tyrosine kinases. Oncogene 38, 151-163

71. Matteucci E, Bendinelli P and Desiderio MA (2009) Nuclear localization of active HGF receptor Met in aggressive MDA-MB231 breast carcinoma cells. Carcinogenesis 30, 937-945

72. Chaudhary SC, Cho MG, Nguyen TT, Park KS, Kwon MH and Lee $\mathrm{JH}$ (2014) A putative pH-dependent nuclear localization signal in the juxtamembrane region of c-Met. Exp Mol Med 46, e119

73. Tauszig-Delamasure S, Yu LY, Cabrera JR et al (2007) The TrkC receptor induces apoptosis when the dependence receptor notion meets the neurotrophin paradigm. Proc Natl Acad Sci U S A 104, 13361-13366

74. Bordeaux MC, Forcet C, Granger L et al (2000) The RET proto-oncogene induces apoptosis: a novel mechanism for Hirschsprung disease. EMBO J 19, 4056-4063

75. Wang H, Boussouar A, Mazelin L et al (2018) The Proto-oncogene c-Kit Inhibits Tumor Growth by Behaving as a Dependence Receptor. Mol Cell 72, 413-425 e415

76. Genevois AL, Ichim G, Coissieux MM et al (2013) Dependence receptor TrkC is a putative colon cancer tumor suppressor. Proc Natl Acad Sci U S A 110, 30173022

77. Luo Y, Kaz AM, Kanngurn S et al (2013) NTRK3 is a potential tumor suppressor gene commonly inactivated by epigenetic mechanisms in colorectal cancer. PLoS Genet 9, e1003552

78. De Oliveira AT, Matos D, Logullo AF et al (2009) MET Is highly expressed in advanced stages of colorectal cancer and indicates worse prognosis and mortality. Anticancer Res 29, 4807-4811

79. Petrelli A, Circosta P, Granziero L et al (2006) Ab-induced ectodomain shedding mediates hepatocyte growth factor receptor down-regulation and hampers biological activity. Proc Natl Acad Sci U S A 103, 5090-5095
80. Pacchiana G, Chiriaco C, Stella MC et al (2010) Monovalency unleashes the full therapeutic potential of the DN-30 anti-Met antibody. J Biol Chem 285, 36149-36157

81. Cignetto S, Modica C, Chiriaco C et al (2016) Dual Constant Domain-Fab: A novel strategy to improve half-life and potency of a Met therapeutic antibody. Mol Oncol 10, 938-948

82. Vigna E, Chiriaco C, Cignetto S et al (2015) Inhibition of ligand-independent constitutive activation of the Met oncogenic receptor by the engineered chemically-modified antibody DN30. Mol Oncol 9, 1760-1772

83. Vigna E, Pacchiana G, Chiriaco C et al (2014) Targeted therapy by gene transfer of a monovalent antibody fragment against the Met oncogenic receptor. J Mol Med (Berl) 92, 65-76

84. Basilico C, Modica C, Maione F, Vigna E and Comoglio PM (2018) Targeting the MET oncogene by concomitant inhibition of receptor and ligand via an antibody-"decoy" strategy. Int J Cancer [Epub ahead of print]

85. Perk LR, Stigter-van Walsum M, Visser GW et al (2008) Quantitative PET imaging of Met-expressing human cancer xenografts with 89Zr-labelled monoclonal antibody DN30. Eur J Nucl Med Mol Imaging 35, 1857-1867

86. Athauda G, Giubellino A, Coleman JA et al (2006) c-Met ectodomain shedding rate correlates with malignant potential. Clin Cancer Res 12 (14 Pt 1), 4154-4162

87. Fu L, Guo W, Liu B et al (2013) Shedding of c-Met ectodomain correlates with c-Met expression in non-small cell lung cancer. Biomarkers 18, 126-135

88. Lv H, Shan B, Tian Z, Li Y, Zhang Y and Wen S (2015) Soluble c-Met is a reliable and sensitive marker to detect c-Met expression level in lung cancer. Biomed Res Int 2015,626578

89. Engelman JA, Zejnullahu K, Mitsudomi T et al (2007) MET amplification leads to gefitinib resistance in lung cancer by activating ERBB3 signaling. Science 316, 1039-1043

90. Siebel C and Lendahl U (2017) Notch Signaling in Development, Tissue Homeostasis, and Disease. Physiol Rev 97, 1235-1294 